\title{
ACUTE UNDIFFERENTIATED FEVER IN INTENSIVE CARE UNITS
}

Srikanth Ram Mohan T1ㅡ, Venkatarathnamma P², B. N. Raghavendra Prasad ${ }^{3}$ Beena P. M$^{4}$

\section{HOW TO CITE THIS ARTICLE:}

Srikanth Ram Mohan T, Venkatarathnamma P, B. N. Raghavendra Prasad, Beena P. M. "Acute Undifferentiated Fever in Intensive Care Units". Journal of Evolution of Medical and Dental Sciences 2014; Vol. 3, Issue 11, March 17; Page: 2851-2856, DOI: 10.14260/jemds/2014/2216

ABSTRACT: Acute undifferentiated fever (AUF) is common in tropical regions of the developing world, its specific etiology is often unknown. It's common causes include malaria, dengue fever, enteric fever, leptospirosis, rickettsial infection. AUF is defined as fever without any localised source of infection, of 14 days or less in duration. The objective of the study was to focus on identifying the causes of AUF in patients admitted to Intensive care units \& to determine importance of clinical examination in identifying the cause. It was a prospective study done in our Medical college Hospital at Kolar, Karnataka between 1-11-2010 to 30-11-2011. Cases presenting to hospital aged >18 years with complaints of Fever \& admitted in Intensive care units were included in study. A total of 558 cases were enrolled. The clinical findings were noted and subsequent Investigations required were asked for. The study compromised of approximately equal number of Male \& Female patients \& age varied from 18 - 100 years. There was a clear seasonal variation - More no of cases were admitted between April \& November. Majority presented with Fever of Short duration (1-3 days). Certain well defined syndromes were identified like:

- Fever with Thrombocytopenia - the most common of all the syndromes.

- Fever with Myalgia \& Arthralgia,

- Fever with Hepatorenal dysfunction,

- Fever with Encephalopathy,

- Fever with Pulmonary - Renal dysfunction and

- Fever with Multiorgan dysfunction (MODS).

Out of 558 cases AUF was noted in 339 cases (60.86\%). An etiological diagnosis could be made for 218 cases (39.06\%). Leptospirosis was the commonest cause with 72 cases (12.9\%). The no of cases with Dengue were 48(8.6\%), Malaria -25 (4.4\%), Viral fever -35 (6.2\%), Mixed infections 12 (2.1\%), Pulmonary Tuberculosis -25 ( 4.4\%) and one case of Rickettsial Infection. MODS was the most common presentation in AUF patients, seen in 108 cases (31.8\%) and 40 cases expired.

A study of AUF done at Siriraj Hospital, Bangkok revealed that etiologies of AUF could be determined in $38.7 \%$ of study population. It has close relevance to present study where etiologies were determined in $39.06 \%$ cases. Mortality rates observed were similar.

KEYWORDS: AUF.

INTRODUCTION: Fever is a common presenting complaint in the developing world. Acute undifferentiated febrile illness is common in tropical regions of the developing World, its specific etiology is often unknown, making accurate diagnosis, effective treatment, and targeted public health measures difficult. ${ }^{1}$ The common causes of acute undifferentiated fever include malaria, dengue fever, enteric fever, leptospirosis, rickettsial infection, hanta virus \& Japanese encephalitis. ${ }^{2}$ The definitions which are used commonly are mentioned below. 
ACUTE UNDIFFERENTIATED FEVER (AUF): Fever, without any localized source of infection, of 14 days or less in duration.

LOCALISED FEVER: Fever, with a symptom, sign, or an investigation that localized the source of infection to skin or soft tissue, respiratory, gastrointestinal, or genitourinary systems was defined as a localized fever. Patients having malignancy or auto immune disorder are also included in the group. ${ }^{3}$

CLASSIC PYREXIA OF UNKNOWN ORIGIN: Is defined as temperature more than $38.3^{\circ} \mathrm{C}\left(101^{\circ} \mathrm{F}\right)$, for more than three weeks with no diagnosis after three outpatient visits or after 3 days of inpatient stay in the hospital or one week of invasive ambulatory investigations. ${ }^{4}$

Many cases of AUF in the tropical countries are empirically treated with Anti tubercular, Antimalarial and Antibiotics before being referred to a tertiary care hospital. There is paucity of literature on AUF \& very few studies reported from India. Our study was aimed at identifying the causes of AUF in patients who presented to our institute.

OBJECTIVE: The objective of the study is to focus on identifying the causes of acute undifferentiated fever in patients being admitted to Intensive care units. To determine the importance of clinical examination in identifying the cause of AUF.

MATERIALS \& METHODS: It was a prospective study which was carried out in our Medical college Hospital based at Kolar, Karnataka between 1-11-2010 to 30-11-2011. It is a 850 bedded tertiary care hospital with in house capacity of 13 ICU \& 30 Medical Intensive care beds.

INCLUSION CRITERIA - All cases that presented to our hospital with complaints of Fever in the above mentioned period and admitted in Intensive care units were included in the study. Patients aged greater than 18 years were included.

EXCLUSION CRITERIA: All patients in whom a definite cause or source of infection such as a wound, abscess, Cellulitis, Pelvic inflammatory disease, otitis media, trauma with injuries, burns and chronic infective etiologies like Chronic pancreatitis, Prolonged Bedridden patients with long term urinary catheters \& those with poisoning consumption, Snake bite were excluded from the study.

A total of 557 cases were enrolled based on the study guidelines. Informed written consent was taken from the patient's relatives prior to the enrolment. History was collected from the patient's attendants by the treating Doctors. Majority of the patients who presented to our institute were referred from nearby Hospitals \& only a few presented directly to our Hospital. The information collected from patients was entered into a proforma sheet that consisted of all the clinical findings \& Investigation reports.

The clinical findings were noted and subsequent Investigations required were asked for. All the patients were scrutinized through Basic Investigations such as Complete Blood counts with peripheral blood smear \& ESR, Random Blood Sugar, Renal Function Tests -Blood urea \& Serum creatinine, Chest X ray, Urine Routine Examination, HIV \& ECG. Based on the Requirements other investigations asked for were Serum Electrolytes, Liver Function Tests, Serology For Hepatitis B \&C, 
Dengue \& Leptospirosis serology, Peripheral Smear for Malarial parasite, Rapid card test for Malaria, 2 D ECHO, CT scan Brain \& Chest, CSF For analysis, Blood, Urine \& Stool cultures, Coagulation profile, Arterial Blood gas Analysis.

The comorbidities noted were Hypertension, Diabetes Mellitus, Chronic Obstructive pulmonary disease, Chronic Alcoholism, Steroid abuse, Chronic Liver disease \& Malignancy.

Majority of Patients were treated with a combination of Higher Antibiotics - Third \& fourth Generation Cephalosporins, Quinolones, Macrolides. Antibiotics were changed according to antibiogram based on culture reports. Other supportive measures employed were IV Fluids, Ryles tube Feeds, Stockings application to prevent Deep vein Thrombosis, Nasal oxygen \& Nebulization, Inotropic support, Non-Invasive \& invasive ventilation, Anti edema measures.

Ethical Committee clearance has been taken from the institute.

\section{RESULTS:}

- The study compromised of approximately equal number of Male \& Female patients \& the age group of the cases enrolled varied widely from 18 - 100 years. There was a clear seasonal variation observed - More no of cases were admitted between April \& November. Majority of the patients presented with the complaint of Fever of Short duration (1-3 days). Other complaints noted were Breathlessness, Cough with expectoration, Diarrhoea, Headache \& vomiting, altered sensorium \& restless behavior, pain abdomen, Jaundice \& passing high coloured urine, Decreased urine output.

Some patients had no h/o of Fever but had Hypotension, Tachypnea, Tachycardia, Respiratory distress, altered mental status \& disseminated intravascular coagulation.

Certain well defined syndromes were identified like:

- Fever with Thrombocytopenia - the most common of all the syndromes.

- Fever with Myalgia \& Arthralgia,

- Fever with Hepatorenal dysfunction,

- Fever with Encephalopathy,

- Fever with Pulmonary- Renal dysfunction and Fever with Multiorgan dysfunction (MODS).

- Out of 558 cases enrolled for the study an etiological diagnosis could be made for 218 cases (39.06\%). The cases with proved etiology included Malaria, Dengue, Leptospirosis, Viral Fever, Mixed Infections, Tuberculosis \& Rickettsial infection. Leptospirosis was the most Common identifiable cause with 72 cases that constituted $12.9 \%$ of all the cases. The no of cases with Dengue were 48 cases(8.6\%), Malaria -25 cases (4.4\%), Viral fever -35 cases (6.2\%), Mixed infections -12 (2.1\%), Pulmonary Tuberculosis -25 cases(4.4\%) and one case of Rickettsial Infection. The results are tabulated as follows 


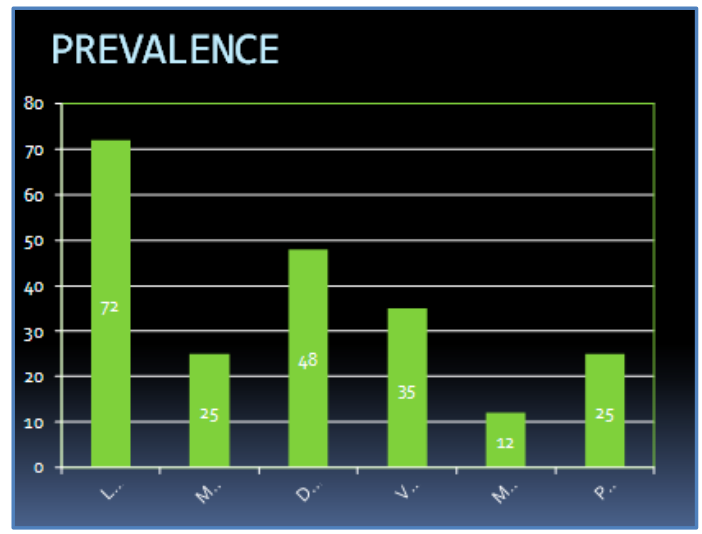

INDEX - L -Leptospirosis, M- Malaria, D-Dengue, V-Viral fever, M - Mixed infections, PPulmonary tuberculosis.

Of the 218 cases with proved etiology 100 cases recovered (45.8\%), 78 cases were lost for follow up \& 40 cases expired.

Analysis of the study revealed that majority of the cases presenting to our institute with complaints of Fever \& admitted in ICU were found to have Acute Undifferentiated Fever. AUF was noted in 339 cases (60.86\%). Etiological Diagnosis was possible in 218 cases that constituted 39.1\% of the study population.

Multi organ Dysfunction was the most common presentation in those cases who satisfied the criteria of AUF. It was seen in 108 of 339 cases (31.8\%) and 40 cases expired.

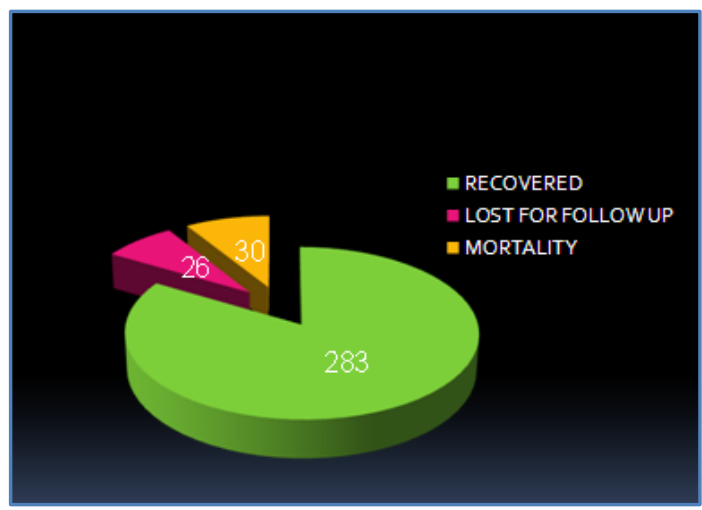

DISCUSSION: Acute undifferentiated Fever is a common problem encountered in tropical countries. The presentation varies widely from being totally stable to a life threatening emergency. Majority of the cases presenting to a tertiary care hospital are treated initially at primary care centres with oral \& intravenous antibiotics, antimalarials.

The complaints of Fever are commonly given less importance. A detailed clinical examination performed carries lot of significance in analyzing the source of infection \& the system primarily involved. The spectrum of the cases presenting to each hospital varies considering the epidemiology of a particular disease in the region. For example, malaria is a common disease that is endemic in many parts of India. The spectrum of cases compromising AUF is dynamic \& ever changing. It depends to a large extent on the endemicity of a particular disease in a region \& the epidemics that occur. 
Good history along with detailed clinical examination would throw light in approaching a clinical diagnosis which can be confirmed by lab investigations \& Imaging studies. However in a country like India where tertiary care facilities are limited, clinical diagnosis is of critical importance. The patients can be screened through a basic panel of investigations such as complete blood counts, Renal function tests, urine routine examination, chest $\mathrm{x}$ ray \& sputum examination.

Here we would like to throw light on a study done by thangarasu et al in 2008 done on 342 cases in Chennai, aimed at designing a protocol for the emergency department management of AUF which concluded implementing a rational, standardized protocol demonstrates a potential to lower rates of unnecessary testing $\&$ antimicrobial use. ${ }^{2}$

A one year prospective study conducted in Christian Medical College Hospital, Vellore, Tamilnadu on 398 Adult hospitalized patients concluded that scrub typhus was the most common cause of AUF compromising $47.5 \%$ of the cases in their study group. ${ }^{5}$

Another study performed on 100 cases in 2011 in Gandhi Medical college, Secunderabad, Andhra Pradesh yielded Dengue as the most common cause constituting 39\% cases followed by Scrub Typhus- $19 \%$ of the cases. The above mentioned two studies highlight the prevalence \& reemergence of Scrub typhus as a cause of AUF in India. Majority of the patients in India don't undergo the Weil Felix Test, though Non- specific is helpful in confirming the diagnosis of Rickettsial Infection. 6

A study of AUF done on 1240 patients in Siriraj Hospital, Bangkok revealed that etiologies of AUF could be determined in 471 cases $-38.7 \%$ of the study population. It has very close relevance to our study in which etiologies could be determined in $39.06 \%$ of the cases. ${ }^{7}$ Mortality rate in both the studies were nearly the same $-1.1 \%$ in Thailand $\& 0.71 \%$ in our study.

\section{REFERENCES:}

1. Mancock SR, Jacobsen KH, Brito de Bravo N, Russell KL, Negrete M, Olson JG et al. Etiology of Acute Undifferentiated Febrile Illness in the Amazon Basin of Ecuador. Am J Trop Med. Hyg. 2009; 15:975-77.

2. Thangarasu S, Natarajan P, Rajavelu P, Rajagopalan A, Devey JSS. A Protocol for the Emergency department Management of Acute undifferentiated Febrile Illness in India. International Journal of Emergency Medicine.2011; 4:57-60.

3. Joshi R, Colford Jr J M, Reingold AL, Kalantri S. Non-Malarial Acute Undifferentiated Fever in a Rural Hospital in Central India: Diagnostic Uncertainty and overtreatment with Anti- Malarial Agents. Am J Trop Med. Hyg. 2008; 78:393-99.

4. Rupali P. Fever of Unknown Origin. In: API Textbook of Medicine. Eds: Munjal YP, Sharma SK, Agarwal AK, Gupta P, Kamath SA, Nadkar MY et al. The Association of Physicians of India. Jaypee Brothers Medical Publishers, Mumbai; 9th ed. 2012: p.42-46.

5. Chrispal A, Boorugu H, Gopinath KG, Chandy S, Prakash JAJ, Thomas EM et al. Acute undifferentiated Febrile Illness in adult Hospitalised patients: the Disease spectrum \& Diagnostic Predictors - an experience from a tertiary care hospital in South India. Trop Doct 2010, 40(4):230-4.

6. Kammili N, Swathi A, Devara SM, Anuradha P R. Prevalence of Scrub Typhus among Acute Undifferentiated Febrile Illness cases Provisionally Diagnosed as Dengue Fever. Journal of Evolution of Medical \& Dental Sciences. 2013; 2: 2661-64. 


\section{ORIGINAL ARTICLE}

7. Leelarasamee A, Chupaprawan C, Chenchittikul M, Udompanthurat S. Etiology of acute undifferentiated febrile illness in Thailand. J Med Assoc Thai.2004; 87: 464-72.

\section{AUTHORS:}

1. Srikanth Ram Mohan T.

2. Venkatarathnamma P.

3. B. N. Raghavendra Prasad

4. Beena P. M.

\section{PARTICULARS OF CONTRIBUTORS:}

1. Final Year Post Graduate, Department of General Medicine, Sri Devaraj Urs Medical College, Kolar.

2. Professor, Department of General Medicine, Sri Devaraj Urs Medical College, Kolar.

3. Professor and HOD, Department of General Medicine, Sri Devaraj Urs Medical College, Kolar.
4. Professor and HOD, Department of Microbiology, Sri Devaraj Urs Medical College, Kolar.

\section{NAME ADDRESS EMAIL ID OF THE} CORRESPONDING AUTHOR:

Dr. Srikanth Ram Mohan T,

Room No. 214,

PG Men's Hostel,

Sri Devaraj Urs Medical College, Kolar.

E-mail: sritadaind04@yahoo.com

Date of Submission: 06/02/2014.

Date of Peer Review: 07/02/2014.

Date of Acceptance: 25/02/2014.

Date of Publishing: 13/03/2014. 